\title{
Design and Implementation of N-Point FFT Processor for MIMO-OFDM Systems using Radix-
} $\mathrm{N}$

\author{
B.Mahalakshmi, B.V.N.Aparna, Ch. Lakshmi Prasanna, E. Anusha, A.B.V.L.N.Jyothi, J.Soundarya, \\ B.Anusha
}

\begin{abstract}
This paper presents Single-path Delay Feedback (SDF) architecture for implementing Fast Fourier Transform (FFT) for Multiple-Input Multiple-Output Orthogonal Frequency Division Frequency Multiplexing (MIMO-OFDM). The architecture of Single-path Delay Feedback and memory scheduling are the basic concepts used to implement the FFT processor with variable length. Depending on the SDF architecture, we implement the FFT processor-based design which is proposed in this paper. In this paper, we use MIMOOFDM high data rates, high efficiency and high throughput. In this paper, we use radix-4 algorithm to implement the sequence because the speed of the operation is high. The functionality verification and the synthesis are carried out by using XLINIX.14.2.
\end{abstract}

Keywords: Single-Path Delay Feedback (SDF), Fast Fourier Transform (FFT), Multiple-Input Multiple-Output (MIMO), Orthogonal Frequency Division Multiplexing (OFDM), Radix-4.

\section{INTRODUCTION}

Fast Fourier Transform (FFT) is mostly used for many applications. By using this, we easily evaluate the Discrete Fourier Transform (DFT). Among many communication applications digital signal processing is one which uses Fast Fourier Transform.

Decimation in time and Decimation in frequency are two different ways to perform Fast Fourier Transform. FFT is the main block in OFDM as it deals many operations to be done.

Revised Manuscript Received on April 13, 2020.

* Correspondence Author

B.Mahalakshmi*, assistant professor in the stream of Electronics and communication engineering, Bapatla Women's Engineering college, Bapatla, Email: mahalakshmibollimuntha@gmail.com

B.V.N.Aparna, Electronics and Communication engineering, Bapatla Women's Enigeering College, Bapatla, Inida. Email: aparnaece2609@gmail.com

Ch.Lakshmi Prasanna, Electronics and Communication engineering, Bapatla Women's Enigeering College, Bapatla, Inida. Email: prasanna19.ece@gmail.com

E.Anusha, Electronics and Communication engineering, Bapatla Women's Enigeering College, Bapatla, Inida. Email: anushaevuru@gmail.com

A.B.V.L.N.Jyothi, Electronics and Communication engineering, Bapatla Women's Enigeering College, Bapatla, Inida. Email: jyothi4499@gmail.com

J.Soundarya, Electronics and Communication engineering, Bapatla Women's Enigeering College, Bapatla, Inida. Email: soundaryajaladi1999@gmail.com

B.Anusha, Electronics and Communication engineering, Bapatla Women's Enigeering College, Bapatla, Inida. Email: bommaanusha13@gmail.com

(c) The Authors. Published by Blue Eyes Intelligence Engineering and Sciences Publication (BEIESP). This is an open access article under the CC BY-NC-ND license (http://creativecommons.org/licenses/by-nc-nd/4.0/)
By using, MIMO the throughput elevated greatly when compared with existing systems. The lifetime of MIMOOFDM systems is high while using in wireless The many applications come under the credit of OFDM are wired communication modems such as digital subscriber lines to wireless communication modems.communications. When we combine MIMO with OFDM, the issues are overcome upon each other. When FFT is combined with MIMO we get the results at high speed. By using the combination of MIMO-OFDM with FFT the results are getting a very fast manner, high reliability, high efficiency and high throughput. For these reasons we use a combination of FFT with MIMO-OFDM.

FFT architectures are different types. They are memorybased, cache memory, sequential, parallel, parallel iterative, array and pipelined architectures. Pipelined architectures helped in a better way for the implementation of FFT.

\section{ANALYSIS LITERATURE}

Fast Fourier Transform is the major block in the Orthogonal Frequency Division Multiplexing systems [5]. OFDM has assigned in a large range of applications from wired communication modems, such as digital subscriber lines to wireless-communication modems, like IEEE802.16 WiMAX or 3GPP Long Term Evaluation (LTE), to process baseband data [5].

Y.G.Li, J.H. Winters and N.R.Sollenberger proposed Multiple-Input Multiple-Output devices, data throughput can be elevated drastically [8]. Hence MIMO-OFDM systems feed data rate and reliability in wireless communication [8]. Without a proper design, the complexity of the FFT processor in MIMO systems increases linearly with the number of data streams [8].

B.G.Jo and M.H.Sunwoo proposed pipeline schemes are the architectures most widely adopted for the implementation of FFT [7]. From the memory access perspective, in-place memory updating schemes perform the computation in the three phases: writing in the inputs, updating intermediate values and reading out the results [7]. In the updating phase, the processor reuses the radix-r processor, such that a single radix-r butterfly is sufficient to complete N-point FFT computation [7]. However, it is nonoverlapping characteristics that make the butterfly idle inmemory write and read phases overall process is lengthy [7].

T.S. Basha and others proposed high speed MDC architectures and memory scheduling are very much suitable

Published By:

Blue Eyes Intelligence Engineering

\& Sciences Publication

(C) Copyright: All rights reserved.

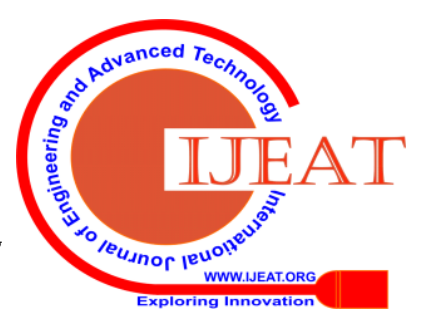


for FFT processor in multiple input multiple output OFDM systems, because the constant multipliers are used to store the twiddle factors instead of ROM, which utilizes $100 \%$ area and reduces the delay [5].

\section{RADIX-4 SDF}

Pipelined architecture is also known as cascaded architecture and is used in most designs. The most commonly used pipelined architectures such as SDC, SDF, MDC and MDF. Pipelined architecture has many advantages compared to other architectures like simplicity, high throughput, fast, small area and energy-efficient implementation.

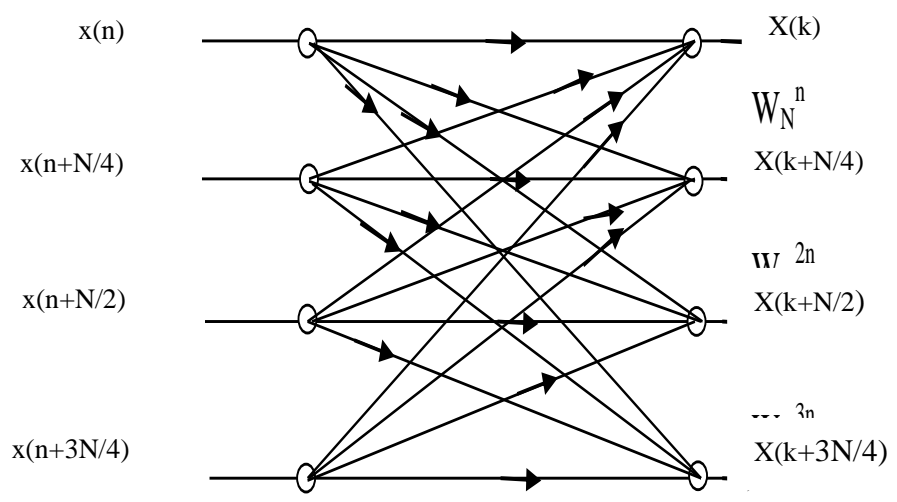

Fig 1 Radix-4 Butterfly Diagram

The Fig 1 represents the radix- 4 butterfly diagram. It contains less number of computations compared to radix-2 algorithm. If we use radix- 4 algorithm, the twiddle factors are decreased for this reason we choose radix-4 algorithm in this paper. Also these are the benefits for radix-4 algorithm.

By choosing this radix-4 algorithm, the hardware utilization is reduced and the complex multiplier is minimized. Radix-2 algorithm utilizes high power, is the major drawback. The fig 1 shows the basic butterfly diagram for radix-4, which is also used for long length of sequences.



Fig 2 Equivalent graph for fig 1

In the name it is clearly, that it takes 4inputs and gives 4outputs. But for radix-2, it considers only 2 inputs and gives 2 outputs. In Radix-4, the every output comes out with the interconnection of all inputs. For example, the first output is comes out due to internal connection of remaining inputs along with main input. The internal operations are done at here only like multiplication, additions, and subtractions. The whole operation is mainly depends upon the twiddle factors. The twiddle factor contains exponent of product of some values like $\mathrm{N}$, m etc. where $\mathrm{N}$ represents the number of stages, $\mathrm{m}$ is the stage index. Also we calculate the twiddle factor by using the combination of sin and cosin with some products which are discussed already in above.

Fig 2 is the equivalent graph for fig1. In this paper, we implement the FFT sequence by using a radix-4 algorithm. The results are very fast, it occupies less area compare radix-2 algorithm. In this paper, we proposed 16 point FFT sequence, for this sequence if we use the radix-2 complexity is increased, to reduce complexity we use the radix-4.

Stage I

stage II

stageIII

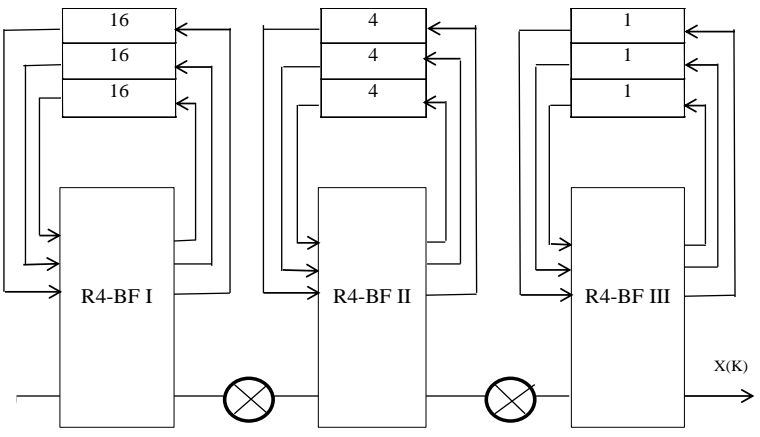

$\mathrm{x}(\mathrm{n})$

Fig 3 Block diagram for 16 points SDF

In Fig 3, we have 3 radix-4 butterflies. Here we consider a 16 point sequence, if we use the radix- 2 algorithm the complexity will increase, for that reason we choose the radix- 4 algorithm. The stages are found by using the $4^{\mathrm{N}}, \mathrm{N}$ represents the number of stages. For 16 point sequence we have used 3 radix -4 butterflies. Single path Delay Feedback (SDF), allows only a single bit at a time and remaining bits are feedback to the input. The main advantage is, there is no Intersymbol interference. The SDF is introduced to overcome the drawback present in the SDC. In SDC, there is no feedback due to this if the output has error we cannot change that. To overcome this drawback we introduce SDF. We also use multipliers; through this the output is sent to the next stage. At this multiplier the operations are performed.

The R4SDF is better than the R2SDF because, it operation performed highly and the results are out in fast manner. The R4SDF is not only used for small sequences, it also used for larges sequences. It is efficient technique compared to R2SDF. .

\section{RESULT AND DISCUSSION}

\section{A.Rtl Schematic:}

The Fig 4 shows the RTL schematic for 16 point radix-4 SDF.

Published By:

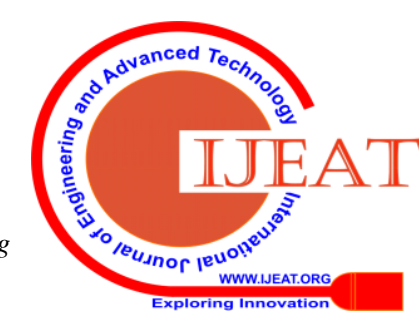




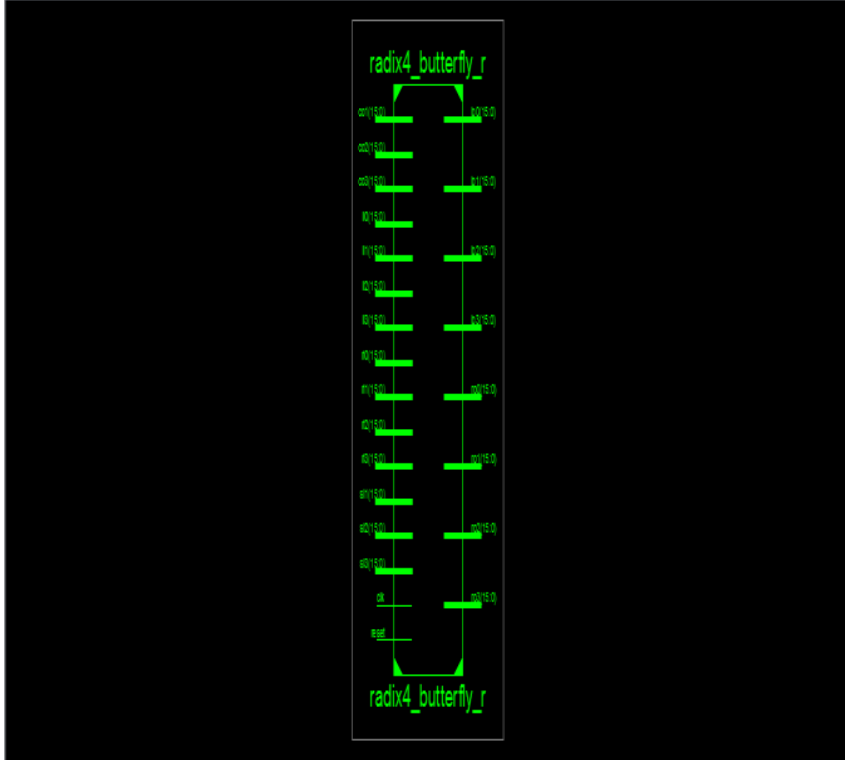

Fig 4 RTL Schematic for 16 points SDF FFT processor

\section{B.Simulation Results:}

The Fig 5 shows the simulation results for radix-4 16 point FFT sequence.

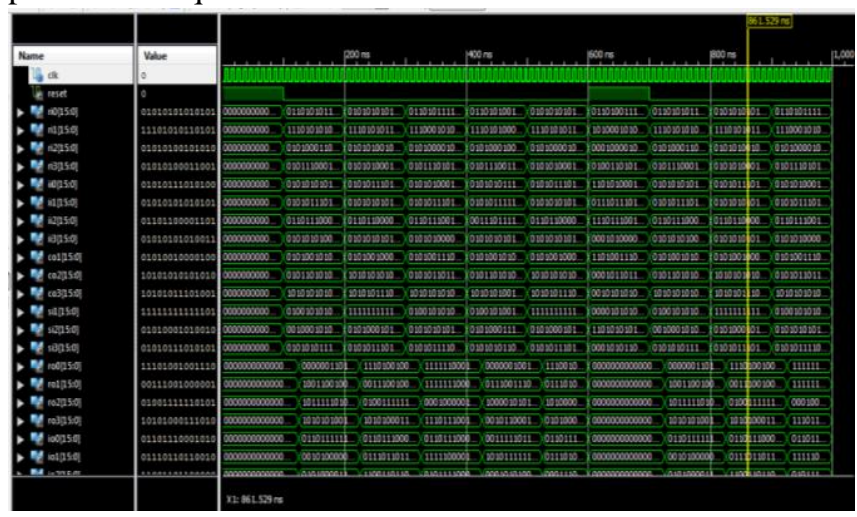

Fig 5 simulation results

\section{C.Design Summary:}

We utilize 768 registers along with 768 flipflops. For the synthesis we have to utilize the devices with the number of slices counts 1584 out of $165 \%$ and the number of flipflops is 848 out of 1920 with $44 \%$ and LUT's counts to 2392 out of 1920 with $124 \%$. The I/O's we use 354 and bounded input-output will be 354 out of 66 with $536 \%$. We use multi $18 * 18$ input outputs 4 out of 4 with $100 \%$ and the generator clock is 1 out of 24 with $4 \%$.

We require a 13.312 ns clock period with a frequency of $75.119 \mathrm{MHz}$ and get the delay of $13.312 \mathrm{~ns}$ with 31 levels of logic. The delay for logic is 6.531 ns with $88.3 \%$ and 0.866 ns with $11.7 \%$. The total real-time to xst completion is $18.00 \mathrm{~s}$ and the total CPU time to xst completion is $17.86 \mathrm{~s}$ and the total memory usage is $251908 \mathrm{~Kb}$.

\section{CONCLUSION}

The proposed high-speed SDF architecture and memory scheduling are very much suitable for the FFT processor in multiple input multiple output OFDM system because the constant multipliers are used to store the twiddle factors, which uses less area and reduces the delay. Further we can improve this method by using mixed-radix we can reduce the computations and complexity and we will get the efficient results in the same as the radix-4.

\section{REFERNCES}

1. P.Anusha, A.Ashok Kumar, "design of a single-path pipelined mixed sdc-sdf architecture for radix-4 FFT", international journal of research, vol *, issue \% , May 2019.

2. Yong Chul Jung, Jae-chan Cho and others, "Area-efficient pipelined FFT processor for zero-padded-signals”, electronics 2019

3. K.Periyarselayam. G.Saravana Kumar, M.Anand, " A novel architecture ofdm radix-4 single-path delay feedback FFT using MCSLA", Indonesian journal of electrical engineering and computer science, vol.10, number 1, april 2018.

4. Mohmad Ali, Naeem Ramzan and others, "efficient FPGA implementation of high-speed throughput mixed-radix multipath delay commutator FFT processor for MIMO-OFDM", AECE, vol 17 number 1, 2017.

5. T. S. Basha and others, "Implementation of high-speed MDC FFT processor for MIMO-OFDM systems", ijareeie, col. 3, issue 9, September 2014

6. Y. W. Lin, C. Y. Lee, "Design of an FFT/IFFT processor for MIMO OFDM systems", IEEE Trans on circuits and systems I, vol.54 number 4,pp.807-815, april 2007.

7. G.Jo and M.H. Sunwoo, "New continuous-flow mixed-radix (CFMR) FFT processor using novel in-place strategy,: IEEE Transactions. Circuits Syt. I, Reg. Papers, vol 52, number 5,pp. 911-919, may 2005.

8. Y G LI, J H Winters, and N. R. Sollenberger, "MIMO OFDM for wireless communications: signal detection with enhanced channe estimation,: IEEE Trans. Communications., vol 50 number 9 pp. 14711477, September 2002.

9. EE. Swartzland, W.K.W Young, and S.J. Joseph, "A radix 4 delay commutator for fast fourier transform processor implementation," IEEE journal of solid state circuits, vol.19, number 5., pp. 702 709,October 1984

10. https://en.m.wikipedia.org/wiki/FastFouriertransform

11. https://en.m.wikipedia.org/wiki/radix

12. https://en/m/wikipedia.org/wiki-MIMO-OFDM

\section{AUTHORS PROFILE}

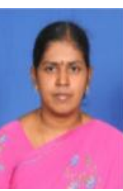

B.Mahalakshmi, M.Tech. Currently working in the stream of Electronics and Communication Engineering in Bapatla Women's Engineering College.

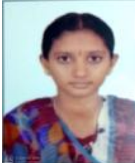

B.V.N.Aparna, pursuing B.Tech in the stream of Electronics and Communication Engineering in Bapatla Women's Engineering College.

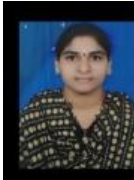

CH. Lakshmi Prasanna, pursuing B.Tech in the stream of Electronics and Communication Engineering in Bapatla Women's Engineering College.

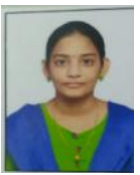

E.Anusha, pursuing B.Tech in the stream of Electronics and Communication Engineering in Bapatla Women's Engineering College.

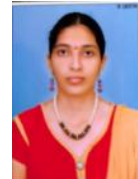

A.B.V.L.NJyothi, pursuing B.Tech in the stream of Electronics and Communication Engineering in Bapatla Women's Engineering College.

Published By:

Blue Eyes Intelligence Engineering \& Sciences Publication 
J.soundarya, pursuing B.Tech in the stream of Electronics and Communication Engineering in Bapatla Women's Engineering College.

B.Anusha, pursuing B.Tech in the stream of Electronics and Communication Engineering in Bapatla Women's Engineering College.

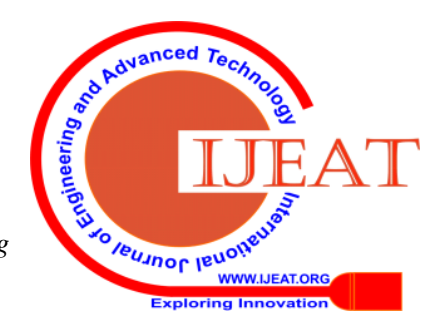

